\title{
eNeuro
}

Research Article: New Research / Disorders of the Nervous System

\section{Agmatine alleviates cisplatin-induced ototoxicity by activating PI3K/AKT signaling pathway}

https://doi.org/10.1523/ENEURO.0434-21.2022

Cite as: eNeuro 2022; 10.1523/ENEURO.0434-21.2022

Received: 13 October 2021

Revised: 18 February 2022

Accepted: 23 February 2022

This Early Release article has been peer-reviewed and accepted, but has not been through the composition and copyediting processes. The final version may differ slightly in style or formatting and will contain links to any extended data.

Alerts: Sign up at www.eneuro.org/alerts to receive customized email alerts when the fully formatted version of this article is published.

Copyright @ 2022 Zhang et al.

This is an open-access article distributed under the terms of the Creative Commons Attribution 4.0 International license, which permits unrestricted use, distribution and reproduction in any medium provided that the original work is properly attributed. 
4 Department of Otolaryngology, the Second Hospital of Hebei Medical University,

5 No.215 Heping West Road, Shijiazhuang 050000, Hebei, China

6

$7 \quad$ * Corresponding author

8 Qiang He

9 Department of Otolaryngology, the Second Hospital of Hebei Medical University,

10 No.215 Heping West Road, Shijiazhuang 050000, Hebei, China

11 Email: qianghe@hb2h.com

12

13

14 Running title: Agmatine alleviates cisplatin-induced ototoxicity

15

16 Funding: The study was supported by the Key Project of Medical Science Research in

17 Hebei Province in 2018 (20180338).

18

19

20

21

22

23

24 


\section{Abstract}

26 Cisplatin-induced ototoxicity can be partially attributed to excessive reactive oxygen

27 species (ROS) production, and agmatine is well-known for the activation of the 28 phosphatidylinositol 3-kinase (PI3K)/ protein kinase B (AKT) pathway to inhibit ROS

29 production. Whether agmatine could be utilized to alleviate cisplatin-induced ototoxicity 30 is investigated. Cisplatin exposed House Ear Institute-Organ of Corti 1 (HEI-OC1) cells 31 and cochlear explants showed increased ROS production detected by 32 2'-7'dichlorofluorescin diacetate (DCFH-DA) staining and decreased cell viability 33 detected by Cell Counting Kit-8 (CCK-8) or Myosin 7a staining, which could be reversed 34 by the agmatine pre-treatment. Cisplatin intraperitoneally injected C57BL/6 mice 35 demonstrated damaged auditory function as indicated by distortion products otoacoustic 36 emissions (DPOAE) and auditory brainstem response (ABR) assays, and 37 trans-tympanically administrated agmatine in the left ears could partly prevent the 38 auditory function loss. Mechanistically, down-regulated B-cell lymphoma 2 (Bcl-2) 39 expression, up-regulated Bcl2 Associated $\mathrm{x}$ (Bax) expression, and diminished p-PI3K and 40 p-AKT expression were detected in cisplatin-exposed HEI-OC1 cells and cochlear 41 explants, which could be prevented by the pre-treatment with agmatine. Our investigation 42 demonstrates that agmatine pre-treatment could alleviate cisplatin-induced ototoxicity 43 with the activation of PI3K/AKT signaling pathway.

45 Keywords: Agmatine; cisplatin-induced ototoxicity; PI3K/AKT 


\section{Significance Statement}

47 The study demonstrated that agmatine pre-treatment could activate PI3K/AKT signaling

48 pathway to alleviate cisplatin-induced ototoxicity.

49

50 


\section{$51 \quad$ Introduction}

52 Cisplatin was approved in 1978 by the US Food and Drug Administration (FDA) to treat

53 ovarian cancer and metastatic testicular patients(Farrell 2015). Although serious side

54 effects such as bone marrow depression, nephrotoxicity, and ototoxicity can occur,

55 cisplatin remains the most widely utilized and available chemotherapeutic drug to treat

56 solid malignant tumors (Glynne-Jones and Hoskin 2007, Chang and Chinosornvatana

57 2010, Johnstone, Suntharalingam et al. 2016, Rottenberg, Disler et al. 2021). The

58 incidence of cisplatin-induced ototoxicity can range from twenty percent to seventy

59 percent, which may manifest with progressive, irreversible, and bilateral hearing loss

60 (Tang, Wang et al. 2021). Young children are more inclined to cisplatin-induced

61 ototoxicity with delayed speech development and psychosocial and cognitive

62 development (Knight, Kraemer et al. 2005, Rybak, Mukherjea et al. 2019).

63 It is generally believed that cisplatin-induced ototoxicity may be attributed to the

64 excessive reactive oxygen species (ROS) production by the cochlea (Yu, Gu et al. 2020),

65 and endoplasmic reticulum stress is a target for treatment of hearing loss (Wang and $\mathrm{Xu}$

66 2020). Multiple promisings strategies have been performed to alleviate, treat, and prevent

67 cisplatin-induced ototoxicity, and none of these strategies has been confirmed or

68 recommended by the FDA (Gentilin, Simoni et al. 2019, Mukherjea, Dhukhwa et al.

69 2020).

70 Among the multiple signaling pathways contributing to the survival and differentiation of

71 hair cells, phosphoinositide-3-kinase (PI3K)/protein kinase B (AKT) pathway is well

72 investigated (Liu, Wei et al. 2021), which might play an essential role in inner ear hair

73 cells survival to resistance against harmful stimuli(He, Zheng et al. 2021). It is worth 
74 noting that, in neonatal cochlear spiral ganglion explants, PI3K/AKT signaling mediates 75 brain-derived neurotrophic factor-induced neurite formation (Mullen, Pak et al. 2012). In 76 the noise-induced cochlea injury, PI3K/AKT pathway activation induced by 77 deferoxamine may promote mesenchymal stem cell homing (Peyvandi, Abbaszadeh et al. 78 2018).

79 Agmatine is formed by L-arginine decarboxylation and hydrolyzation to putrescine, 80 which can bind to N-methyl-D-aspartate (NMDA) receptors and $\alpha 2$-adrenergic receptors 81 to function as novel neurotransmitters and neuromodulators (Regunathan 2006, Piletz, 82 Aricioglu et al. 2013). Substantial preclinical and initial clinical evidence has indicated 83 the possibility to treat opioid addiction, mood disorders, neurotrauma, neurodegenerative 84 diseases, and cognitive disorders (Xu, Gao et al. 2018, Akasaka and Fujiwara 2020).

85 This investigation utilizes agmatine to treat cisplatin exposed HEI-OC1 cells, cochlear 86 explants, or cisplatin affected mice and finds that agmatine alleviates hearing loss with 87 reduced ROS production and cell loss and up-regulated PI3K/AKT signal pathway. 88 Therefore, as an adjuvant drug, agmatine has the potential value in reducing ototoxicity 89 caused by cisplatin chemotherapy.

90

91 Methods \& materials

92 Cell viability

93 HEI-OC1 cells (5, 000/well) were seeded in 96-well plates in three replicates, and 94 relevant agmatine $(10,50,100,200 \mu \mathrm{M})$ and or cisplatin $(5,10,30,50 \mu \mathrm{M})$ were 95 incubated for indicated hours. CCK-8 (Dojindo Laboratories) was added to each well 96 with the final concentration of $10 \%$ for 4 hours. The optical density values were 
97 measured at $450 \mathrm{~nm}$ with a Bio-Rad plate reader.

\section{Cisplatin exposed HEI-OC1 cells culture}

99 HEI-OC1 cell line was pre-treated with $100 \mu \mathrm{M}$ agmatine for 2 hours and then cotreated 100 with $30 \mu \mathrm{M}$ cisplatin for 24 hours in appropriate conditions $\left(33^{\circ} \mathrm{C}, 5 \% \mathrm{CO}_{2}\right.$, high-glucose 101 DMEM medium, 5\% fetal bovine serum, Gibco).

\section{$102 \underline{\text { ROS detection }}$}

103 2'-7'dichlorofluorescin diacetate (DCFH-DA) working solution (10 $\mu \mathrm{M}$, Beyotime, 104 S0033) was added into six-well plates and incubated the plates at $37^{\circ} \mathrm{C}$ for 30 minutes. 105 After the incubation, the fluorescence was observed with the LEXT OLS5100 laser 106 scanning confocal microscope.

\section{Cisplatin exposed cochlear explants}

108 Cochleae from C57BL/6 mice (three days postnatal) were dissected out and seeded intact 109 on Cell-Tak (BD Biosciences, Franklin Lakes, NJ, USA) coated glass coverslips, which 110 were further incubated with DMEM/F12 medium supplemented with $1 \times$ N2/B27 as 111 recommended by the manufacturer (Invitrogen, Waltham, MA USA) at $37^{\circ} \mathrm{C}$ with $5 \%$ $112 \mathrm{CO}_{2}$. Agmatine $(100 \mu \mathrm{M})$ was used to pre-treat cochlear explants for 2 hours, and then 113 cisplatin $(30 \mu \mathrm{M})$ was added to induce the ototoxicity for 24 hours.

\section{Western blot}

115 HEI-OC1 or cochleae explant lysates were separated by $12 \%$ sodium dodecyl 116 sulfate-polyacrylamide gel and transferred to polyvinylidene fluoride membranes, which 117 was further blocked with 5\% nonfat dry milk and incubated with primary antibodies 118 against GAPDH, Bax, Bcl2, p-PI3K, p-AKT, PI3K, and AKT (Santa Cruz). 119 Peroxidase-conjugated secondary antibody (Sigma-Aldrich, 1:1000 dilution, 2 hours, at 
120 room temperature) was added, and an ECL system (Sigma-Aldrich) was utilized to obtain

121 the signal. The intensity of protein bands was quantified with Image J software. GAPDH

122 was utilized as the loading control to normalize the relative expression.

\section{$123 \quad$ Cisplatin exposed mice}

124 C57BL/6 male mice (four-week-old) purchased from Peking Vital River Laboratory 125 Animal Ltd. (Beijing, China) were maintained. Agmatine $(10 \mu \mathrm{M}, 5 \mu \mathrm{L})$ was 126 trans-tympanically injected into the left ears, while the same volume phosphate-buffered 127 saline (PBS) was injected into the contralateral ears. Then cisplatin $(30 \mathrm{mg} / \mathrm{kg})$ was i.p. 128 administered two hours later. Seven days post cisplatin administration, the auditory 129 brainstem responses (ABR) and distortion product otoacoustic emission (DPOAE) 130 measurements were done. All the procedure was approved by the Ethics Committee of 131 the Second Hospital of Hebei Medical University.

\section{$132 \underline{\text { ABR test }}$}

133 ABR assessment was performed as previously reported (McLean, Clamp et al. 2021). 134 Briefly, anesthetized mice (25 mg/kg xylazine sodium and $100 \mathrm{mg} / \mathrm{kg}$ ketamine, i.p.) 135 were kept warm during the ABR recordings process (highest intensity of acoustic stimuli, $13690 \mathrm{~dB}$ SPL; decrements, $5 \mathrm{~dB}$ SPL) at $38^{\circ} \mathrm{C}$ on the thermostatic heating pad. The hearing 137 threshold at five frequencies $(4,8,16,24$, and $32 \mathrm{kHz})$ was detected with TDT System III 138 apparatus (Tucker Davies Technologies).

\section{$139 \underline{\text { DPOAE test }}$}

140 DPOAE was performed as previously reported with a TDT-RZ6 system (Tucker-Davis 141 Technologies) (Li, Zhou et al. 2021). Two sine wave tones with different frequencies but 142 equal intensities ( $\mathrm{F} 2=1.2 \mathrm{~F} 1, \mathrm{~F} 2$ ranging from 4 to $40 \mathrm{kHz}$ ) were utilized with $1 \mathrm{~s}$ duration 
143 to elicit DPOAE. Twenty adjacent frequency bins around the distortion product 144 frequency were averaged as the surrounding noise floor. DPOAE threshold was 145 determined when the signal was over $5 \mathrm{~dB}$ sound pressure level and over two standard 146 deviations above the surrounding noise floor.

\section{$147 \quad \underline{\text { Immunofluorescence }}$}

148 Intact cochleae were separated from the temporal bone, which were embedded in Optimal 149 Cutting Temperature O.C.T medium (Richard-Allan Scientific, Kalamazoo, MI), snap 150 frozen in liquid nitrogen, and stored at $-80{ }^{\circ} \mathrm{C}$ until use. Five-micrometer sections were 151 cut by a cryostat (Microm HM525, Walldorf, Germany). After being fixed with 4\% 152 paraformaldehyde for $10 \mathrm{~min}$, the sections were blocked with $10 \%$ normal goat serum 153 and permeabilized with $0.3 \%$ Triton $\mathrm{X}-100$ for $2 \mathrm{~h}$ at room temperature. The Fast 154 ImmunoCytoChemistry Staining Kit (Protein Biotechnologies), anti-Myosin 7a antibody 155 (Proteus Bioscience, 25-6790), and DAPI were utilized for hair cell detection.

\section{$156 \quad$ Statistical Analysis}

157 The difference between groups was assessed using one or two-way ANOVA analysis 158 before corresponding post hoc tests. The significance level was set as $p$-value $<0.05$. All 159 statistical analyses were performed with GraphPad Prism (GraphPad Software).

\section{Results}

162 Agmatine alleviates cisplatin-induced ototoxicity in HEI-OC1 cells

163 To optimize the dose of cisplatin, different concentrations of cisplatin $(0,5,10,30$, or 50 $164 \mu \mathrm{M}$ ) were utilized to treat HEI-OC1 cells for 24 hours, and the cell viability was analyzed 165 by CCK-8 assay. Cisplatin (at a dose greater than $30 \mu \mathrm{M}$ ) can markedly reduce cell 
166 viability (Figure 1a). While as to agmatine, only $200 \mu \mathrm{M}$ agmatine could diminish the

167 viability of HEI-OC1 cells (Figure 1b), which indicated that the dose under $200 \mu \mathrm{M}$ was

168 safe. To determine the protective effect of agmatine on cisplatin-induced ototoxicity,

169 HEI-OC1 cells were pre-treated with different concentrations of agmatine for 2 hours and

170 then cotreated with $30 \mu \mathrm{M}$ cisplatin for 24 hours. A significant dose-dependent protective

171 effect was observed, and $100 \mu \mathrm{M}$ agmatine showed the maximal protective effect (Figure

172 1c). These results testified that agmatine could protect HEI-OC1 cells viability upon

173 cisplatin exposure, and $100 \mu \mathrm{M}$ agmatine and $30 \mu \mathrm{M}$ cisplatin were chosen in the

174 following experiment.

175 Agmatine alleviates cisplatin-induced ROS in HEI-OC1 cells

176 We examined ROS production with a mitochondria-specific ROS indicator, DCFH-DA,

177 to determine whether agmatine could alleviate cellular oxidative stress. Cisplatin-induced

178 up-regulated ROS production, while the pre-treatment with agmatine could diminish

179 ROS induction (Figure 2a and 2b). It was worth noting that agmatine alone could not 180 induce the production of ROS. All of these indicated the protection effect caused by 181 agmatine.

$182 \quad$ Agmatine alleviates cisplatin-induced cochleae cell apoptosis in vitro with up-regulated

183 PI3K/AKT pathway

184 In order to decipher the relevant pathway for apoptosis, Bcl-2 family proteins expression 185 was detected with Western blot in cisplatin exposed cells. Elevated Bax (pro-apoptotic, 186 Figure 3a and 3b) and decreased Bcl-2 (anti-apoptotic, Figure 3a and 3c) were observed, 187 which could be reversed by the pre-treatment of agmatine. At the same time, the 188 upstream PI3K/AKT signaling pathway molecules were detected. After cisplatin 
treatment, down-regulated p-PI3K (Figure 3a and 3d) and p-AKT expression (Figure 3a

190 and 3e) were detected. As expected, agmatine administration could up-regulate the $191 \mathrm{PI} 3 \mathrm{~K} / \mathrm{AKT}$ pathway as indicated by the up-regulation of p-PI3K and p-AKT. These data 192 demonstrated that agmatine had the ability to inhibit cisplatin-induced apoptosis with 193 up-regulated PI3K/AKT signaling pathway in HEI-OC1 cells.

\section{Agmatine alleviates cisplatin-induced cochleae explants apoptosis}

195 Myosin 7a staining showed that cisplatin treatment could lead to the conspicuous loss of 196 mature hair cells in the apex (data not shown), basal turns (data not shown), and the 197 middle turn of cochlea. The most significant damage effect was observed on the middle 198 turn of the cochlea, which could be alleviated by the pre-treatment of agmatine (Figure 4a 199 and 4b). Accumulation of ROS may lead to the apoptosis of hair cells. No 200 DCFH-DA-positive cells were detected in the untreated or the agmatine-treated cochlear 201 explants (Figure 4c). While significantly increased DCFH-DA-positive cells were 202 detected after cisplatin exposure, this increase was reversed by agmatine pre-treatment 203 (Figure $4 \mathrm{c}$ and $4 \mathrm{~d}$ ).

204 Agmatine stimulates PI3K/AKT signaling to inhibit the apoptosis in cochleae explant 205 induced by cisplatin exposure

206 Cochleae explant was further utilized to confirm the apoptosis induced by cisplatin 207 exposure. Increased Bax expression (pro-apoptotic, Figure 5a and 5b) and decreased $208 \mathrm{Bcl}-2$ expression (anti-apoptotic, Figure 5a and 5c) were observed in cisplatin exposed 209 cochleae explant, which could be reversed by the agmatine pre-treatment. At the same 210 time, down-regulated p-PI3K (Figure 5a and 5d) and p-AKT expression (Figure 5a and 2115 e) were detected after cisplatin treatment. As expected, agmatine administration could 
212 up-regulate the PI3K/AKT pathway. All of these data confirmed that agmatine could

213 stimulate PI3K/AKT signaling pathway to inhibit cisplatin-induced intrinsic apoptosis

214 pathway in cochleae explant and HEI-OC1 cells.

\section{Agmatine prevents auditory function loss in cisplatin exposed mice}

216 ABR and DPOAE measurements were utilized to indicate the auditory function. Hearing 217 thresholds were significantly elevated at all frequencies tested 14 days after cisplatin 218 exposure, whereas pre-treatment with agmatine could diminish the thresholds (Figure 6a 219 and 6b). All of these indicated that agmatine could partially prevent auditory function 220 loss in cisplatin exposed mice.

221

\section{Discussion}

223 In order to minimize cisplatin-induced ototoxicity, it is vital to find an appropriate 224 strategy to prevent auditory function loss or restore auditory function (Tang, Wang et al. 225 2021). Cisplatin exposed HEI-OC1 cells, cochleae explant, and mice are utilized in this 226 investigation, and we confirm that agmatine alleviates cisplatin-induced ototoxicity with 227 up-regulated PI3K/AKT signaling pathway. Agmatine supplement may help to reduce 228 cisplatin-induced ototoxicity in clinic.

229 Cisplatin can transport into cochlea cells and retain for months to years to undergo 230 hydrolysis to form highly reactive aqua cisplatin complexes, which can induce hair cells 231 apoptosis, inflammation, and permanent hearing loss (Rybak, Mukherjea et al. 2019). Our 232 results demonstrate the beneficial effect of agmatine pre-treatment on cisplatin-induced 233 cochleae cells apoptosis with the inhibition of the downstream mitochondrial apoptotic 234 pathway, thereby protecting cochleae cells from cisplatin-induced ototoxicity in the acute 
235 phase. A long-term effect of agmatine administration should be performed in future 236 investigations.

237 Cisplatin-induced ototoxicity usually appears in the early stages after exposure to 238 cisplatin, primarily affecting the high frequencies and leading to progressive, permanent, 239 and cumulative hearing loss. As indicated in previous reports, DPOAE (Breglio, Rusheen 240 et al. 2017) and ABR (Rybak, Husain et al. 2000) are dysregulated in cisplatin-affected 241 mice. Our investigation testifies that agmatine could improve the degenerative auditory 242 responses ranging from $4 \mathrm{kHz}$ and $32 \mathrm{kHz}$.

243 Multiple intracellular signaling pathways, including PI3K/AKT pathway, can 244 phosphorylate Bad (serine-136) to inhibit apoptosis (Wang and Youle 2009, Lu and 245 Imlay 2021, Muri and Kopf 2021). Our present study demonstrates that agmatine could 246 increase phosphorylation of PI3K and AKT diminished by cisplatin exposure. The 247 activation of PI3K/AKT may contribute to the anti-ototoxicity effect of agmatine on 248 cisplatin exposure.

249 The antioxidant effect of agmatine may act as a scavenger against ROS in human 250 neuronal-like SH-SY5Y cells to maintain mitochondrial membrane potential (Condello, 251 Currò et al. 2011). In RAW 264.7 cells, agmatine has antioxidant activity against 252 lipopolysaccharides-induced ROS accumulation via the activation of PI3K/Akt 253 pathway (Chai, Luo et al. 2016). It is worth noting that agmatine has anti-inflammatory 254 effects, effectively inhibiting the transcription factor NF-אB (Li, Zhu et al. 2020). As to 255 the safe dose identified in our study ( $200 \mu \mathrm{M}$ agmatine), a preprint paper indicates that 256 the safe dose of agmatine can reach to $10 \mathrm{mM}$ (Park, Lee et al. 2020). Such discrepancy 257 may need further detailed analysis. 
258 In summation, we demonstrate that agmatine significantly affects the protection against

259 cisplatin-induced ototoxicity by inhibiting ROS production and mitochondrial apoptosis.

260 Our findings further indicate that agmatine can function as a therapeutic or preventive 261 agent in cisplatin-induced ototoxicity.

\section{Conclusions}

264 Agmatine can be utilized to alleviate cisplatin-induced ototoxicity with up-regulated 265 PI3K/AKT signaling.

267 Declaration of conflict of interest

268 None.

270 References

271 Akasaka, N. and S. Fujiwara (2020) The therapeutic and nutraceutical potential of 272 agmatine, and its enhanced production using Aspergillus oryzae. Amino Acids 273 52(2):181-197.

274 Breglio, A. M., A. E. Rusheen, E. D. Shide, K. A. Fernandez, K. K. Spielbauer, K. M. 275 McLachlin, M. D. Hall, L. Amable and L. L. Cunningham (2017) Cisplatin is retained in the 276 cochlea indefinitely following chemotherapy. Nat Commun 8(1):1654.

277 Chai, J., L. Luo, F. Hou, X. Fan, J. Yu, W. Ma, W. Tang, X. Yang, J. Zhu, W. Kang, J. Yan and H. 278 Liang (2016) Agmatine Reduces Lipopolysaccharide-Mediated Oxidant Response via 279 Activating PI3K/Akt Pathway and Up-Regulating Nrf2 and HO-1 Expression in 280 Macrophages. PLoS One 11(9):e0163634.

281 Chang, K. W. and N. Chinosornvatana (2010) Practical grading system for evaluating 282 cisplatin ototoxicity in children. J Clin Oncol 28(10):1788-1795.

283 Condello, S., M. Currò, N. Ferlazzo, D. Caccamo, J. Satriano and R. Ientile (2011) 
284 Agmatine effects on mitochondrial membrane potential and NF-KB activation protect 285 against rotenone-induced cell damage in human neuronal-like SH-SY5Y cells. J 286 Neurochem 116(1):67-75.

287 Farrell, N. P. (2015) Multi-platinum anti-cancer agents. Substitution-inert compounds for 288 tumor selectivity and new targets. Chem Soc Rev 44(24):8773-8785.

289 Gentilin, E., E. Simoni, M. Candito, D. Cazzador and L. Astolfi (2019) Cisplatin-Induced 290 Ototoxicity: Updates on Molecular Targets. Trends Mol Med 25(12):1123-1132.

291 Glynne-Jones, R. and P. Hoskin (2007) Neoadjuvant cisplatin chemotherapy before 292 chemoradiation: a flawed paradigm? J Clin Oncol 25(33):5281-5286.

293 He, Y., Z. Zheng, C. Liu, W. Li, L. Zhao, G. Nie and H. Li (2021) Inhibiting DNA methylation 294 alleviates cisplatin-induced hearing loss by decreasing oxidative stress-induced 295 mitochondria-dependent apoptosis via the LRP1-PI3K/AKT pathway. Acta Pharmaceutica 296 Sinica $B$.

297 Johnstone, T. C., K. Suntharalingam and S. J. Lippard (2016) The Next Generation of 298 Platinum Drugs: Targeted Pt(II) Agents, Nanoparticle Delivery, and Pt(IV) Prodrugs. Chem $299 \operatorname{Rev} 116(5): 3436-3486$.

300 Knight, K. R., D. F. Kraemer and E. A. Neuwelt (2005) Ototoxicity in children receiving 301 platinum chemotherapy: underestimating a commonly occurring toxicity that may 302 influence academic and social development. J Clin Oncol 23(34):8588-8596.

303 Li, X., J. Zhu, L. Tian, X. Ma, X. Fan, L. Luo, J. Yu, Y. Sun, X. Yang, W. Tang, W. Ma, J. Yan, X. $304 \mathrm{Xu}$ and H. Liang (2020) Agmatine Protects Against the Progression of Sepsis Through the 305 Imidazoline 12 Receptor-Ribosomal S6 Kinase 2-Nuclear Factor-kB Signaling Pathway. Crit 306 Care Med 48(1):e40-e47.

307 Li, Y., X. Zhou and X. Shen (2021) Esketamine may be an ideal substitute for ketamine 308 during cochlear function measurement. Braz J Med Biol Res 54(11):e11503.

309 Liu, Y., M. Wei, X. Mao, T. Chen, P. Lin and W. Wang (2021) Key Signaling Pathways 310 Regulate the Development and Survival of Auditory Hair Cells. Neural Plasticity $3112021: 5522717$.

312 Lu, Z. and J. A. Imlay (2021) When anaerobes encounter oxygen: mechanisms of oxygen 313 toxicity, tolerance and defence. Nat Rev Microbiol. 
314 Mclean, T., P. Clamp, L. Campbell, A. Hampson, S. Chambers, A. Collins, C. Bester and S. 315 O'Leary (2021) The Effect of Different Round Window Sealants on Cochlear Mechanics 316 Over Time. Otol Neurotol 42(8):1253-1260.

317 Mukherjea, D., A. Dhukhwa, A. Sapra, P. Bhandari, K. Woolford, J. Franke, V. Ramkumar 318 and L. Rybak (2020) Strategies to reduce the risk of platinum containing antineoplastic 319 drug-induced ototoxicity. Expert Opin Drug Metab Toxicol 16(10):965-982.

320 Mullen, L. M., K. K. Pak, E. Chavez, K. Kondo, Y. Brand and A. F. Ryan (2012) Ras/p38 and $321 \mathrm{PI3K} /$ Akt but not Mek/Erk signaling mediate BDNF-induced neurite formation on 322 neonatal cochlear spiral ganglion explants. Brain research 1430:25-34.

323 Muri, J. and M. Kopf (2021) Redox regulation of immunometabolism. Nat Rev Immunol $32421(6): 363-381$.

325 Park, E., S. H. Lee, H. Lee, Y.-C. Kim, H. H. Jung and G. J. Im (2020) Protective effect of 326 agmatine against cisplatin-induced apoptosis in an auditory cell 327 line.2020.2003.2024.005314.

328 Peyvandi, A. A., H. A. Abbaszadeh, N. A. Roozbahany, A. Pourbakht, S. Khoshsirat, H. H. 329 Niri, H. Peyvandi and S. Niknazar (2018) Deferoxamine promotes mesenchymal stem cell 330 homing in noise-induced injured cochlea through PI3K/AKT pathway. Cell Prolif $33151(2): \mathrm{e} 12434$.

332 Piletz, J. E., F. Aricioglu, J. T. Cheng, C. A. Fairbanks, V. H. Gilad, B. Haenisch, A. Halaris, S. 333 Hong, J. E. Lee, J. Li, P. Liu, G. J. Molderings, A. L. Rodrigues, J. Satriano, G. J. Seong, G. 334 Wilcox, N. Wu and G. M. Gilad (2013) Agmatine: clinical applications after 100 years in 335 translation. Drug Discov Today 18(17-18):880-893.

336 Regunathan, S. (2006) Agmatine: biological role and therapeutic potentials in morphine 337 analgesia and dependence. Aaps j 8(3):E479-484.

338 Rottenberg, S., C. Disler and P. Perego (2021) The rediscovery of platinum-based cancer 339 therapy. Nat Rev Cancer 21(1):37-50.

340 Rybak, L. P., K. Husain, C. Morris, C. Whitworth and S. Somani (2000) Effect of protective 341 agents against cisplatin ototoxicity. Am J Otol 21(4):513-520.

342 Rybak, L. P., D. Mukherjea and V. Ramkumar (2019) Mechanisms of Cisplatin-Induced 343 Ototoxicity and Prevention. Semin Hear 40(2):197-204. 
344 Tang, Q., X. Wang, H. Jin, Y. Mi, L. Liu, M. Dong, Y. Chen and Z. Zou (2021)

345 Cisplatin-induced ototoxicity: Updates on molecular mechanisms and otoprotective 346 strategies. Eur J Pharm Biopharm 163:60-71.

347 Wang, C. and R. J. Youle (2009) The role of mitochondria in apoptosis*. Annu Rev Genet 348 43:95-118.

349 Wang, Y. and Z. Xu (2020) Endoplasmic reticulum stress as target for treatment of 350 hearing loss. STEMedicine 1(3):e21.

351 Xu, W., L. Gao, T. Li, A. Shao and J. Zhang (2018) Neuroprotective Role of Agmatine in 352 Neurological Diseases. Curr Neuropharmacol 16(9):1296-1305.

353 Yu, D., J. Gu, Y. Chen, W. Kang, X. Wang and H. Wu (2020) Current Strategies to Combat 354 Cisplatin-Induced Ototoxicity. Front Pharmacol 11:999.

355

356 
$357 \quad$ Figure legends

358 Figure 1. The viability of HEI-OC1 cells with the treatment of designed concentration of 359 cisplatin (a), agmatine (b) and different concentration of agmatine and $30 \mu \mathrm{M}$ cisplatin 360 for 24 hours. Data were represented as mean \pm SD. $\mathrm{n}=3$. $* P<0.05, * * P<0.01$ 361 compared with control group; \#P<0.05, \#\#P<0.01 compared with $30 \mu \mathrm{M}$ cisplatin only 362 group.

364 Figure 2. The intracellular level of reactive oxygen species (ROS) in HEI-OC1 cells was detected with 2',7'-Dichlorodihydrofluorescein diacetate (DCFH-DA) staining. a, represent fluorescent images from different groups. b, fluorescence intensity was measured with Image $\mathbf{J}$ software. Data were represented as mean \pm SD. $\mathrm{n}=3$. $* * P<0.01$, $* * * P<0.001$ compared with control group; \#\#P $<0.01$ compared with cisplatin group. 369 Scale bar $=50 \mu \mathrm{m}$.

371 Figure 3. Effect of agmatine on the Bcl-2 family proteins expressions and PI3K/AKT 372 signaling activation in HEI-OC1 cells. Representative Western blot images (a) and 373 quantitative analysis of Bax (b), Bcl-2 (c), p-PI3K (d), and p-AKT (e). Data were 374 represented as mean \pm SD. $\mathrm{n}=3 .{ }^{*} P<0.05,{ }^{* *} P<0.01, * * * P<0.001$ compared with 375 control group; \#\#P $<0.01$ compared with cisplatin group.

377 Figure 4. Effects of agmatine on cisplatin-induced hair cell loss (a) and ROS level (c) in 378 the middle turns of the cochleae explant. Quantification of myosin 7a-positive (b) and 379 DCFH-DA-positive (d) hair cells. Scale bar $=20 \mu \mathrm{m}$. Data were represented as mean \pm 
380 SD. $\mathrm{n}=6$ for each group. $* P<0.05, * * P<0.01$ compared with control group; $\# P<0.05$,

$381 \# \# P<0.01$ compared with cisplatin group.

382

383 Figure 5. Effect of agmatine on the Bcl-2 family proteins expression and PI3K/AKT

384 signaling pathway activation in the middle turns of the cochleae explant. Representative 385 Western blot images (a) and quantitative analysis of Bax (b), Bcl-2 (c), p-PI3K (d), and 386 p-AKT (e). Data were represented as mean \pm SD. $\mathrm{n}=3$. $* * P<0.01, * * * P<0.001$

387 compared with control group; \#\#P $<0.01$, \#\#\#P $<0.001$ compared with cisplatin group.

388

389 Figure 6. Agmatine prevents auditory function loss in cisplatin exposed mice. ABR (a)

390 and DPOAE (b) thresholds were analyzed. Data were represented as mean \pm SD. $n=6$

391 for each group. $* P<0.05, * * P<0.01$ compared with control group; $\# P<0.05$ compared 392 with cisplatin group.

393 

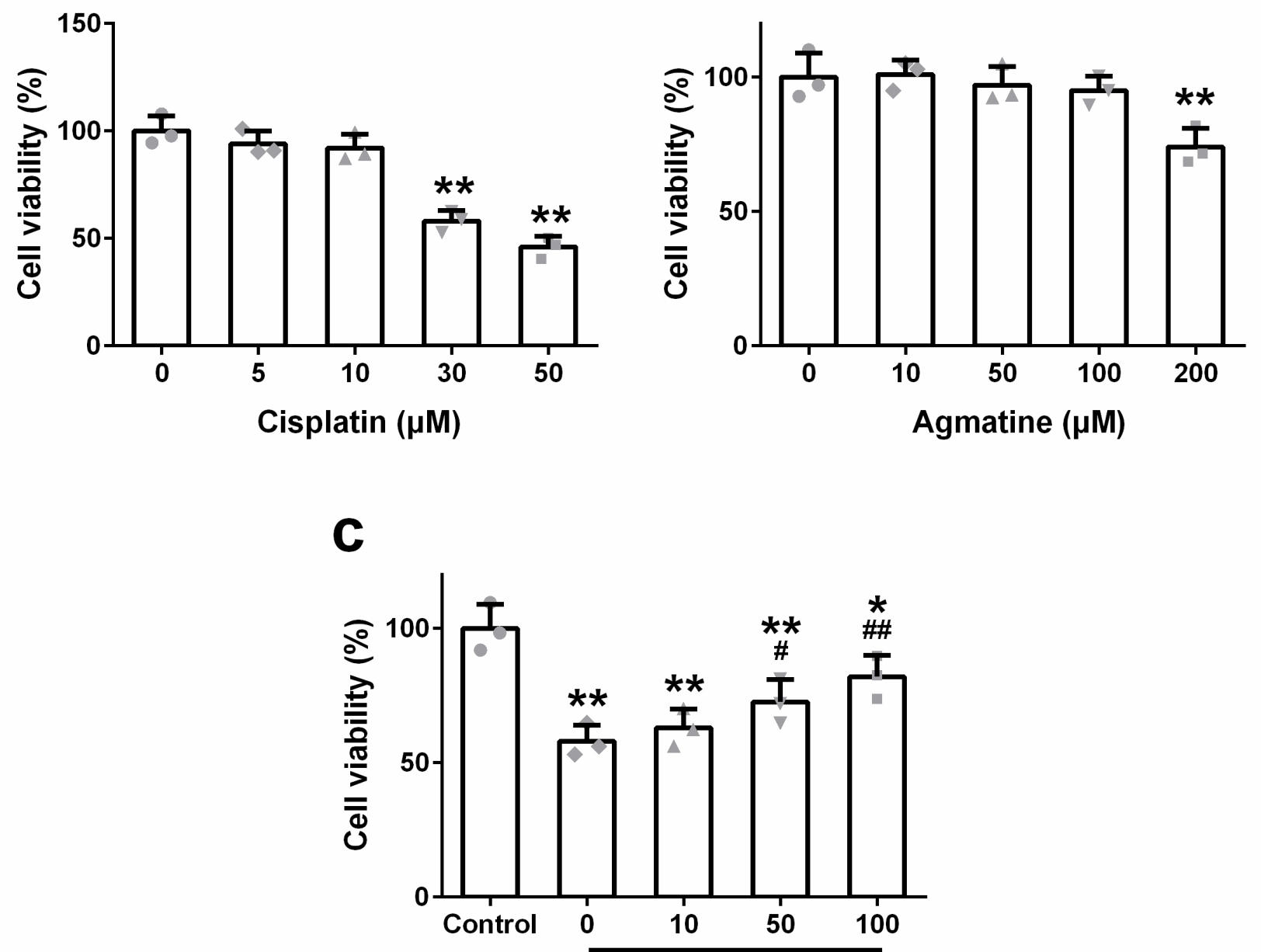

Agmatine $(\mu \mathrm{M})+30 \mu \mathrm{M}$ Cisplatin 


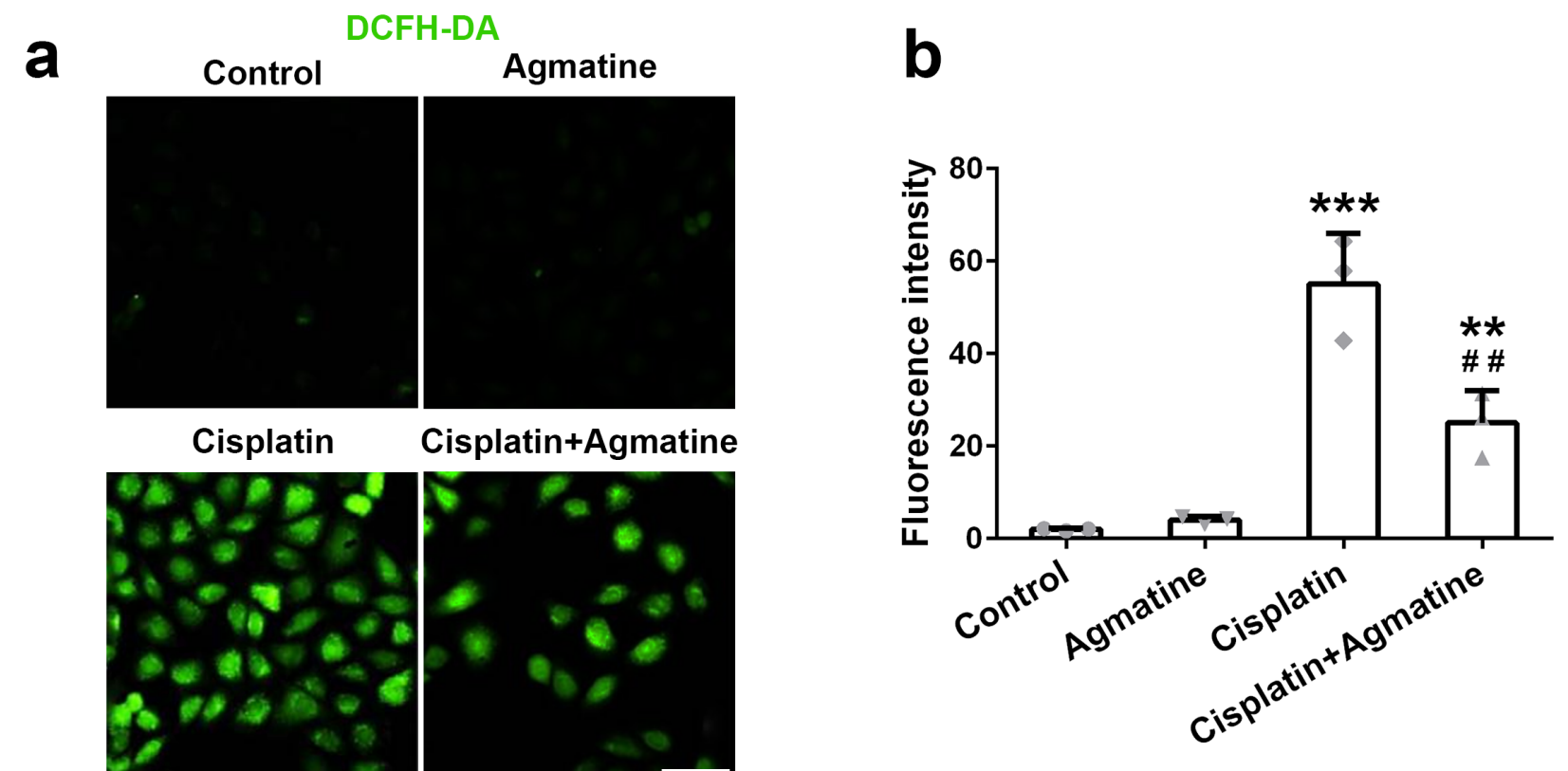


a
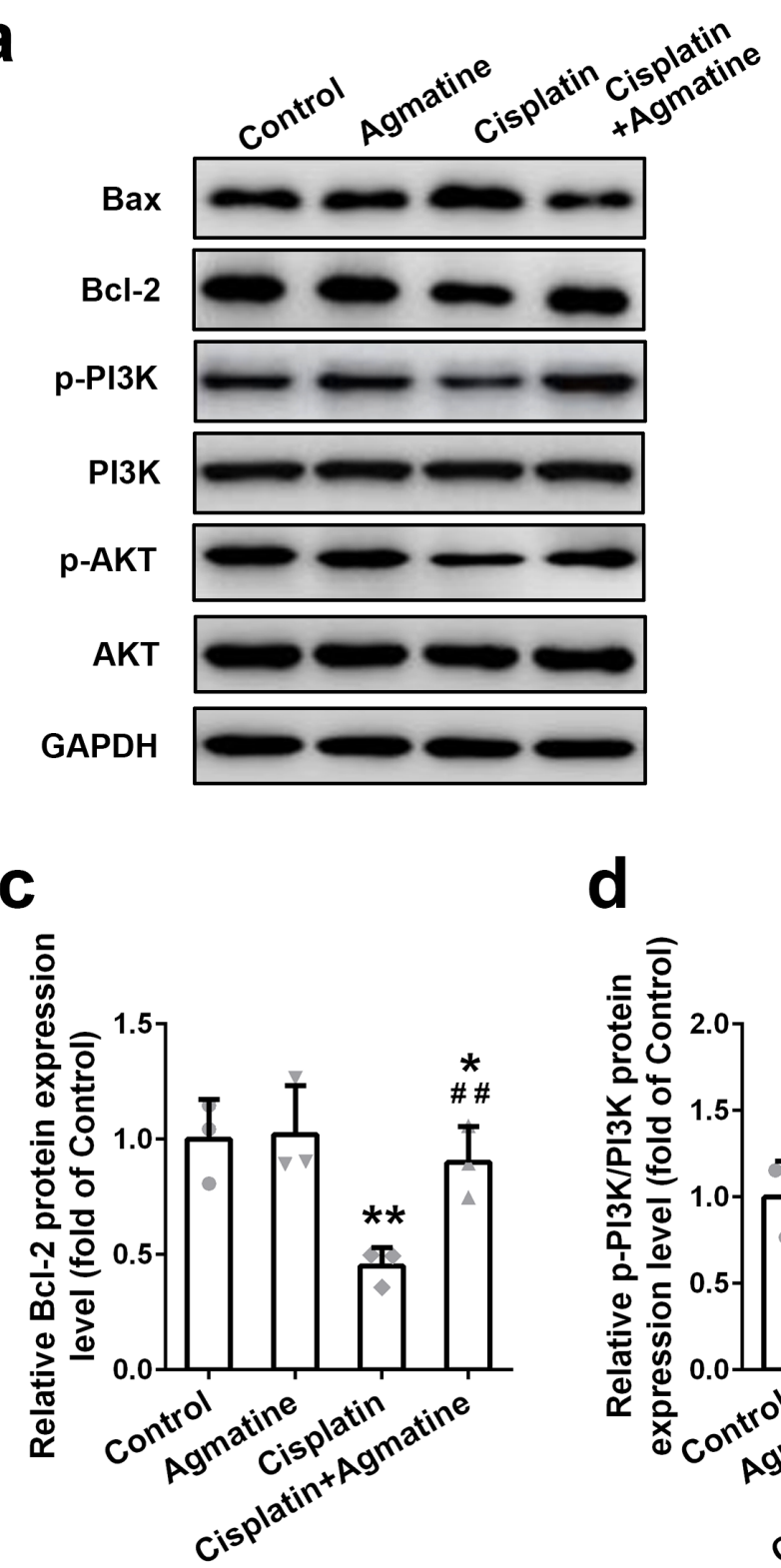

b

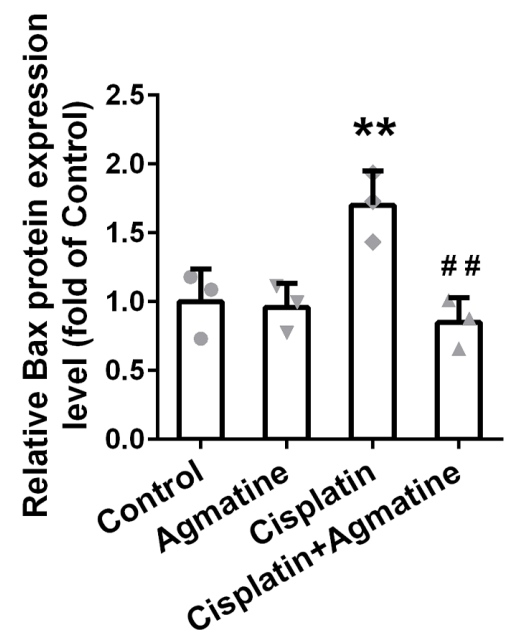

e

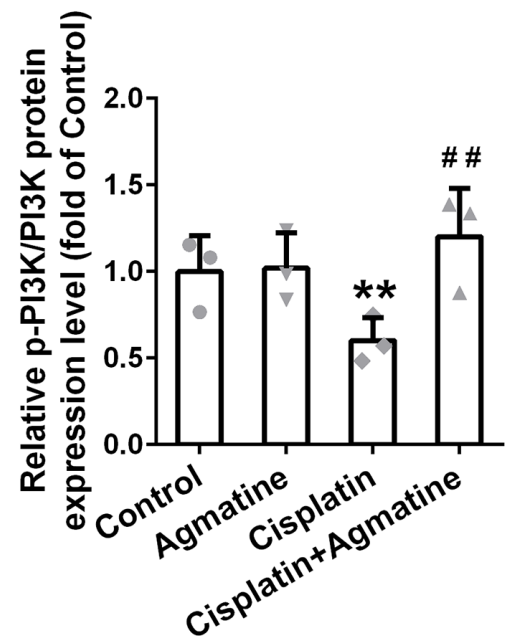

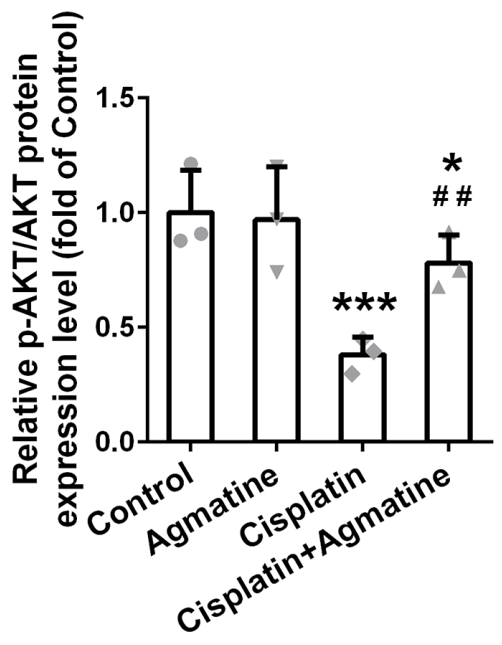



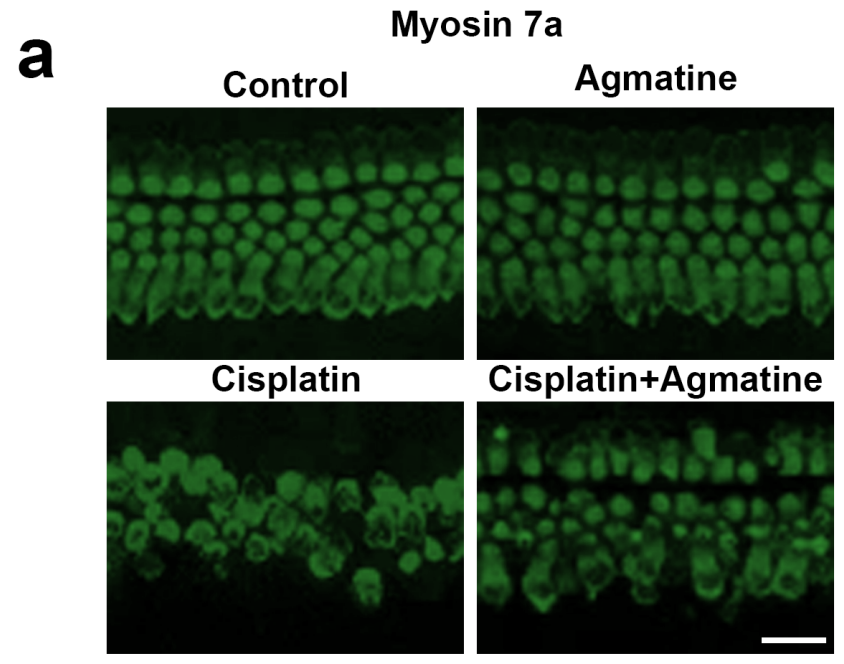

C

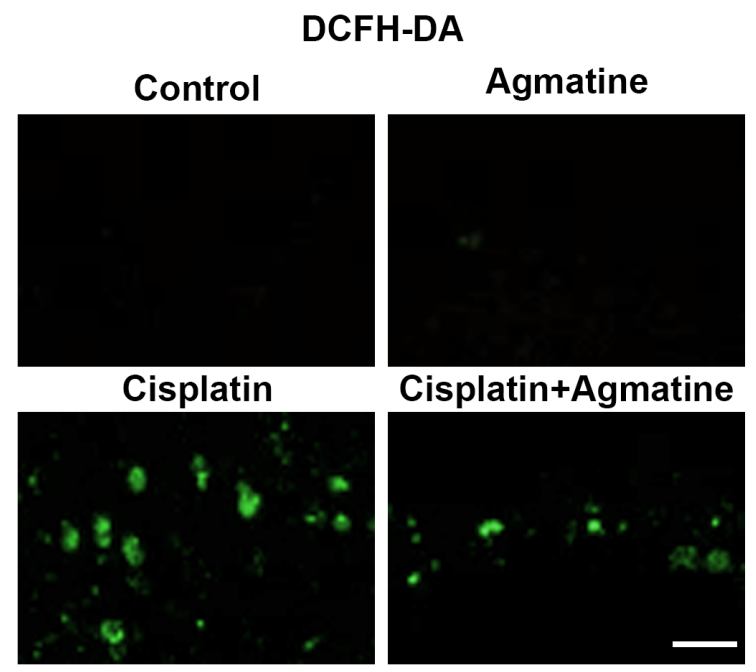

b

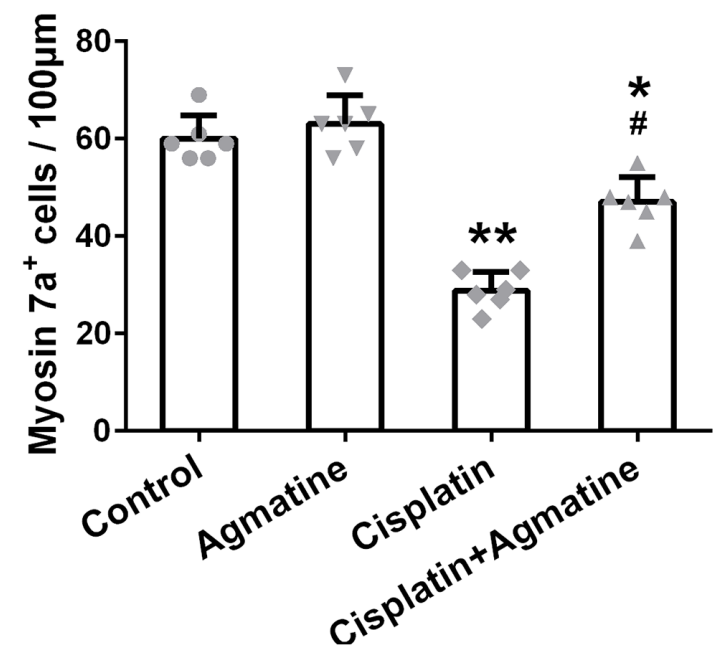

d

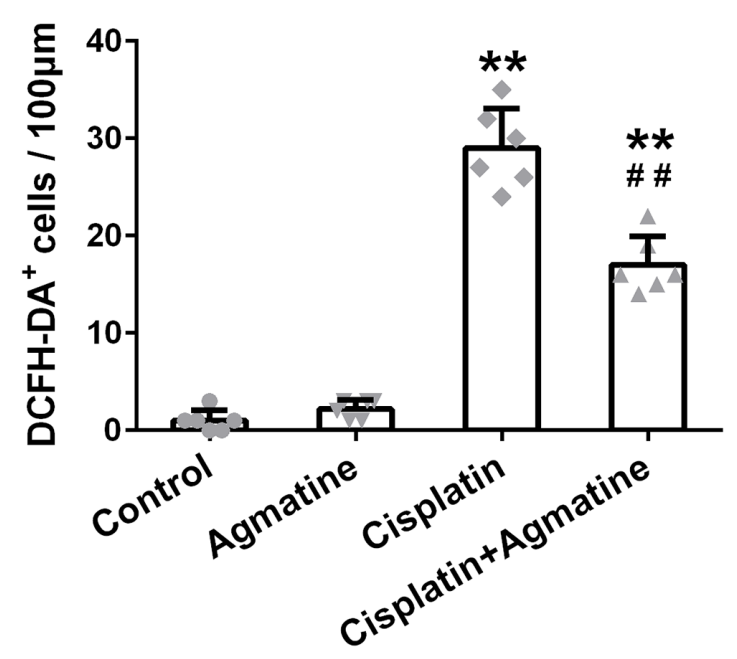


a
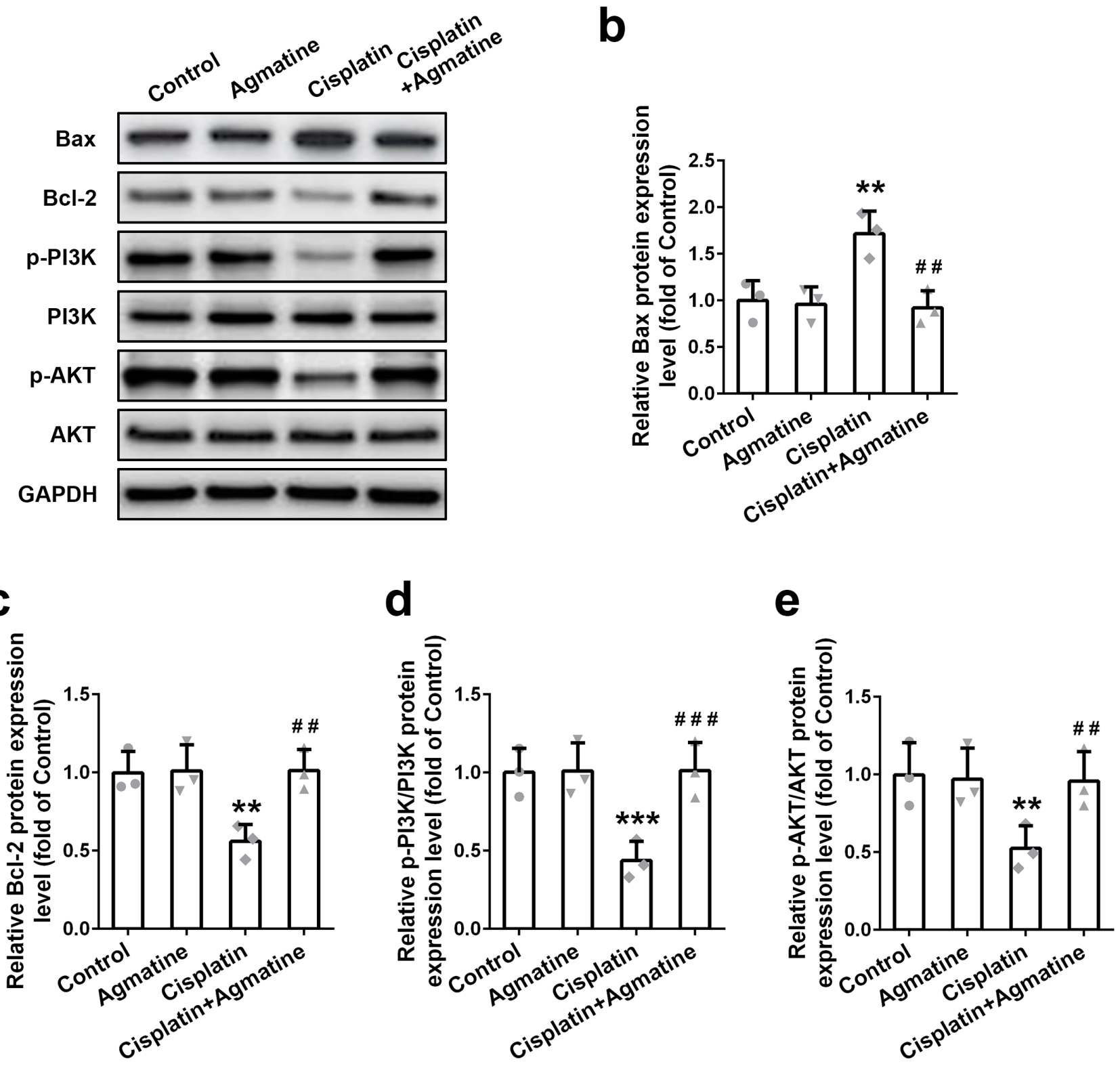

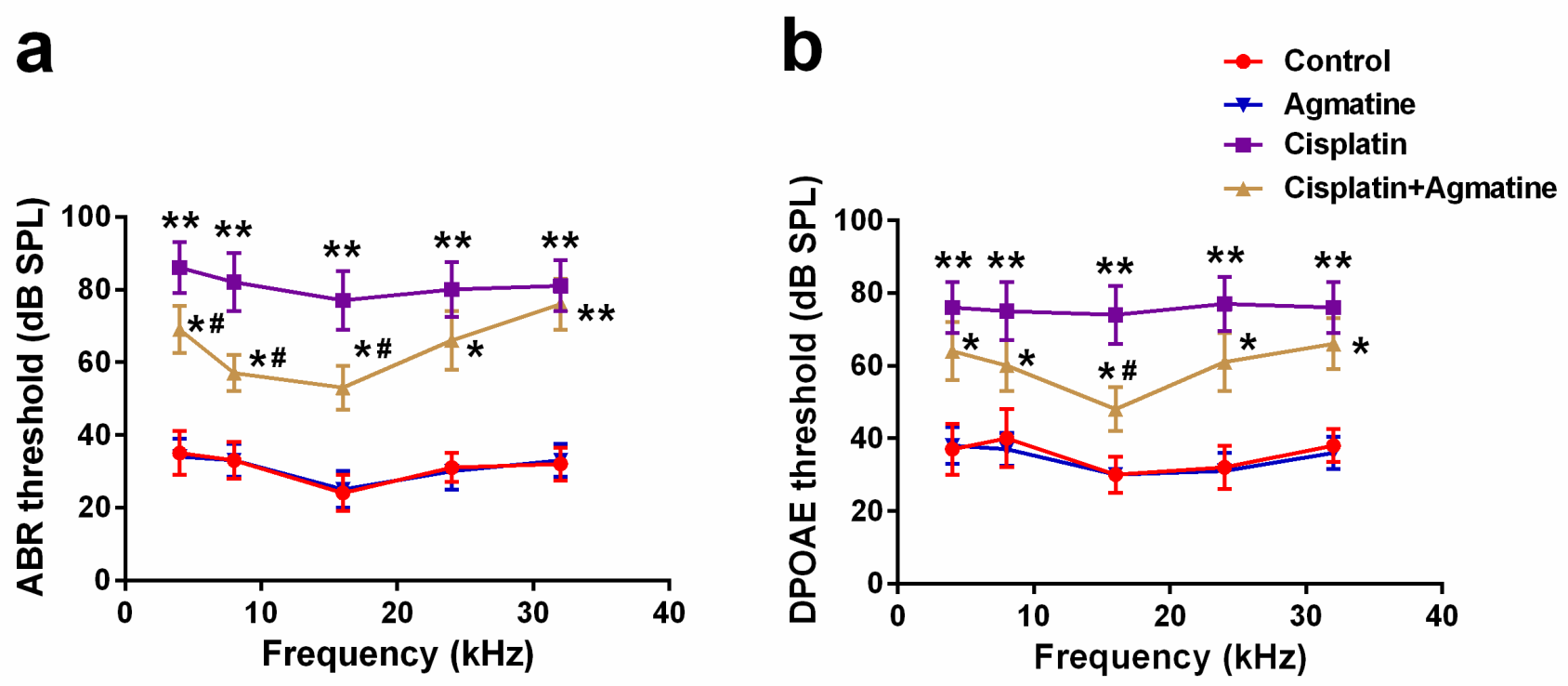